\title{
Sequences of the second internal transcribed spacer of ribosomal DNA for three species of Trichostrongylus (Nematoda: Trichostrongylidae) from sheep in Russia
}

\author{
D. N. KUZNETSOV, N. A. KUZNETSOVA ${ }^{1}$
}

Centre of Parasitology, A. N. Severtsov Institute of Ecology and Evolution, Russian Academy of Sciences, 119049 , Moscow, Mytnaya str., 28, Russia, E-mail: dkuznetsov@mail.ru; ${ }^{1} \mathrm{~V}$. A. Engelgardt Institute of Molecular Biology, Russian Academy of Sciences, 117334, Moscow, Vavilov str., 32, Russia

\begin{abstract}
Summary
For the first time, DNA sequence data were obtained for three species of Trichostrongylus from Russia. Internal transcribed spacer (ITS-2) of ribosomal DNA was sequenced for $T$. axei, $T$. colubriformis and T. probolurus from sheep from the Moscow region. ITS-2 rDNA length was estimated as 238 nucleotides for $T$. colubriformis and $T$. probolurus and 237 nucleotides for $T$. axei. The $\mathrm{G}+\mathrm{C}$ content of the ITS-2 sequences of T. colubriformis, T. axe $i$ and T. probolurus were $31 \%, 32 \%$ and $34 \%$ respectively. The level of interspecific differences in ITS-2 of rDNA of $T$. axei, T. probolurus and T. colubriformis ranged from 3 to 4 $\%$. The ITS- 2 sequences from the Russian specimens were compared with those of T. axei, T. probolurus and T. colubriformis from Australia and Germany. Intraspecific variation ranged from $0 \%$ in $T$. colubriformis to $3.0 \%$ in T. axei.
\end{abstract}

Key words: Trichostrongylus spp.; ITS-2; interspecific differences; intraspecific variation; sheep; Russia

\section{Introduction}

Trichostrongyle nematodes are widespread in mammals (artiodactyles, tylopoda, rodents, lagomorphs, primates) in many countries throughout the world, including Russia (Asadov, 1960; Govorka et al., 1988; Pryadko, 1976; Skrjabin et al., 1954). Infection of the gastrointestinal tract by trichostrongyle nematodes frequently causes a detrimental effect to cattle breeding because of the impact on productivity slowdown in adults and young animals' survivability (Akbaev et al., 1998; Boev et al., 1963; Skrjabin \& Petrov; 1964). Quite often the same host can harbour several species of trichostrongyle nematodes, which differ in some details of their life cycle (Boev et al., 1963; Ivashkin et al., 1989). Reliable species differentiation of Trichostrongylus spp. is essential in effective control of these parasites. Spe- cific differentiation based on morphology is quite secure for trichostrongyle males but not possible for females and eggs. Existing methods of differentiation based on larvae morphology are still quite laborious (Hoste et al., 1995, 1998; McMurtry et al., 2000).

It has been shown that the second internal transcribed spacer (ITS-2) of ribosomal DNA is a valuable target region for species differentiation within the genus Trichostrongylus because of a lack or low levels of intraspecific variation compared with the level of interspecific sequence differences (Chilton et al., 1998; Heise et al., 1999; Hoste et al., 1993, 1995; Wimmer et al., 2004). However, there is no DNA data available for Trichostrongylus spp. found in the territory of Russia. The study of ITS-2 rDNA data for Trichostrongylus spp. from Russia is adding new data to the knowledge of this rDNA domain in trichostrongyle nematodes and can reveal fixed genetical differences. The aim of the present study was to compare the ITS-2 sequences of species of Trichostrongylus from ruminants from the territory of Russia with those previously published from other regions of Europe and from Australia.

\section{Material and Methods}

Male worms of Trichostrongylus axei (Cobbold, 1879), $T$. colubriformis Giles, 1892 and T. probolurus (Railliet, 1896) were removed from the abomasum of a spontaneously infected sheep originating from a private farm in Moscow region. The material was not exposed to any preservative solution. Nematodes were examined using a microscope and identified according to the key of Ivashkin et al. (1989). A single specimen of each species was used. Genomic DNA was isolated from nematodes using the procedure as described by Dallas et al. (2000), but with minor changes. Each worm was placed in $100 \mu \mathrm{l}$ of 10 mM-Tris-Hcl, 0.1 mM-EDTA, pH 8.0, containing $20 \mu \mathrm{g} /$ 
ml-proteinase $\mathrm{K}$ in Eppendorf ${ }^{\mathrm{TM}} 0.5 \mathrm{ml}$ tubes. Samples were incubated in a thermostat for $18 \mathrm{~h}$ at $55^{\circ} \mathrm{C}$. Then the homogenate was heated at $95^{\circ} \mathrm{C}$ for $10 \mathrm{~min}$ and stored at $20^{\circ} \mathrm{C}$.

The ITS-2 was amplified by polymerase chain reaction (PCR) using primers NC1 (forward; 5'-ACGTCTGGTT CAGGGTTGTT-3') and NC2 (reverse; 5'-TTAGTTTCT TTTCCTCCGCT-3') (Gasser et al., 1993). PCR was performed using Sileks $\mathrm{M}^{\mathrm{TM}}$ DNA-amplification kit (Moscow, Russia) in a $25 \mu \mathrm{l}$ reaction volume according to the manufacturer's protocol. PCR was performed according to the scheme: $1^{\text {st }}$ step - denaturation of the DNA at $90^{\circ} \mathrm{C}$ for $2 \mathrm{~min}, 2^{\text {nd }}$ step - denaturation of the DNA at $90^{\circ} \mathrm{C}$ for $15 \mathrm{~s}$, $3^{\text {rd }}$ step - annealing of the primers at $55^{\circ} \mathrm{C}$ for $15 \mathrm{~s}, 4^{\text {th }}$ step - extension of the chain at $72^{\circ} \mathrm{C}$ for $30 \mathrm{~s}, 5^{\text {th }}$ step - exten- sion of the chain at $72^{\circ} \mathrm{C}$ for $5 \mathrm{~min}$. Steps $2^{\text {nd }}$ to $4^{\text {th }}$ were repeated 30 times. PCR products were detected using electrophoresis on $1 \%$ agarose gel. The DNA isolation from the agarose gel was carried out using Sileks $M^{\mathrm{TM}}$ DNAisolation kit according to the manufacturer's protocol.

The ITS- 2 of each worm was sequenced twice in both directions using $\mathrm{ABI}$ "Prism"TM automatic sequencer. The sequences alignment was conducted using the Clustal V program (DNAstar ${ }^{\mathrm{TM}}$ computer application package).

The ITS-2 sequences for three Trichostrongylus species from Russia were compared with those of T. axei from bison in Australia (Hoste et al., 1995) and from ruminants in Germany (Heise et al., 1999), T. colubriformis from sheep in Australia (Hoste et al., 1993) and from ruminants in Germany (Heise et al., 1999), and T. probolurus from

\begin{tabular}{|c|c|c|c|c|}
\hline & 1 & 20 & 40 & 60 \\
\hline & $\mid--$ & $--1--$ & $--1-$ & --1 \\
\hline T.axei & $\mathrm{AATC}$ & ГGGCT & TGTCG & ATA \\
\hline T.axei* & $\cdots$ & $\cdots$ & $\cdots \cdots$ & $\cdots$ \\
\hline T.axei** & $\cdots$ & $\cdots$ & $\cdots \cdots$ & $\cdots$ \\
\hline T.colubriformis & $\cdots$ & $\ldots$ & $\ldots \ldots$ & $\ldots$ \\
\hline T.colubriformis * & $\cdots$ & $\cdots$ & $\cdots \cdots$ & $\cdots$ \\
\hline T.colubriformis ** & $\ldots$ & $\ldots$ & $\ldots$. & $\cdots$ \\
\hline T.probolurus & $\cdots$ & $\cdots$ & $\cdots \cdots$ & $\cdots$ \\
\hline T.probolurus* & $\cdots$ & $\cdots$ & $\cdots$ & $\cdots$ \\
\hline & 61 & 80 & 100 & 120 \\
\hline & $\mid--$ & $--\mid--$ & $--\mid--$ & --1 \\
\hline T.axei & TTG' & TCAGI & $\mathrm{GCAAC}$ & $-\mathrm{AA}$ \\
\hline T.axei* & $\ldots$ & . T... & $\cdots \cdots$ &.- \\
\hline T.axei** & $\cdots$ & . T... & $\cdots \cdots$ & $-\ldots$ \\
\hline T. colubriformis & $\ldots$ & $\cdots \cdots$ & $\cdots \cdots$ & $\mathrm{T}$. \\
\hline T.colubriformis* & $\cdots$ & $\cdots \cdots$ & $\cdots \cdots$ & $\mathrm{T}$. \\
\hline T. colubriformis * * & $\ldots$ & $\cdots \cdots$ & $\cdots \cdots$ & $\mathrm{T}$. \\
\hline T.probolurus & $\ldots$ & $\ldots \ldots$ & $\ldots \ldots$ & $\mathrm{T}$. \\
\hline T.probolurus* & $\cdots$ & $\cdots \cdots$ & $\cdots \cdots$ & T. \\
\hline & 121 & 140 & 160 & 180 \\
\hline & $\mid--$ & $--\mid--$ & $--\mid--$ & --1 \\
\hline T.axei & CGAC & TATGA & $\mathrm{CACTG}$ & TGA \\
\hline T.axei* & $\ldots$ & $\ldots$. & $\cdots \cdots$ & $\cdots$ \\
\hline T.axei** & $\cdots$ & $\cdots \cdots$ & $\ldots \ldots$ & . . \\
\hline T. colubriformis & $\mathrm{T} \ldots$ & $\cdots \cdots$ & T... & $\cdots$ \\
\hline T.colubriformis* & $\mathrm{T} \ldots$ & $\cdots \cdots$ & Т... & $\cdots$ \\
\hline T. colubriformis ** & T. & $\cdots$ & $\mathrm{T} \ldots \mathrm{R}$ & $\cdots$ \\
\hline T.probolurus & $\cdots$ & $\cdots$ & $\cdots \cdots$ & $\cdots$ \\
\hline T.probolurus * & $\cdots$ & $\cdots$ & $\cdots$ & $\cdots$ \\
\hline & 181 & 200 & 220 & 240 \\
\hline & $\mid--$ & $--\mid--$ & $--\mid--$ & --1 \\
\hline T.axei & ATT' & $\mathrm{ACAGG}$ & AATGT & $\mathrm{AT}$ \\
\hline T.axei* & $\cdots$ & $\ldots$ & $\ldots \ldots$ & . \\
\hline T.axei** & $\ldots \mathrm{C}$ & $\cdots$ & $\cdots$ & $\cdots$ \\
\hline T.colubriformis & $\cdots$ & $\cdots \cdots$ & $\cdots \cdots$ & . \\
\hline T.colubriformis* & $\cdots$ & $\cdots \cdots$ & $\cdots \cdots$ & $\cdots$ \\
\hline T. colubriformis ** & $\cdots$ & $\cdots$ & $\cdots \cdots$ & \\
\hline T.probolurus & & $\ldots$ & $\cdots$ & \\
\hline T.probolurus* &. $\mathrm{G}$. & $\mathrm{G} \ldots$ & $\ldots \ldots$ & \\
\hline
\end{tabular}

Fig. 1. Alignment of the ITS-2 sequences (5' to 3') of T. axei, T. colubriformis and T. probolurus according to our results, Hoste et al. (1995) for T. axei*, Hoste et al. (1993) for T. colubriformis*, Chilton et al. (1998) for T. probolurus*, Heise et al. (1999) for T. axei** and T. colubriformis**. (.) - same base as T. axei sequence, (-) - base deletion 
sheep in Australia (Chilton et al., 1998).

The sequence of rodent parasite T. retortaeformis (Hoste et al., 1995) was used as outgroup in the phylogenetic analysis. The dendrogram was generated using program PAUP 4.0b 10 (Swofford, 1998) using maximum parsimony algorithm (MP).

Sequences obtained in the course of this study were deposited in the NCBI GenBank: T. axei - EF427622; T. probolurus - EF427623; and T. colubriformis - EF427624.

\section{Results}

The ITS-2 sequences for three Trichostrongylus species are shown in Fig. 1. The length of ITS-2 rDNA fragments was 238 nucelotides for T. colubriformis and T. probolurus and 237 nucleotides for $T$. axei.

The $\mathrm{G}+\mathrm{C}$ content of the ITS-2 sequences of $T$. colubriformis, T. axei and T. probolurus were $31 \%, 32 \%$ and 34 $\%$ respectively.

The ITS-2 fragments for three Trichostrongylus species studied identical in $92 \%$ : the sequences coincided for 218 out of 238 nucleotides. Five basic regions of interspecific homology (positions 1 - 67, 93 - 117, 134 - 144, 199 211 and 213 - 239; Fig. 1) can be distinguished. In most cases variable positions demonstrated single base substitutions (point mutations). Single base deletion was reported for positions 118, 145 and 156 .

Nine of the point mutations involved substitutions between pyrimidines (i.e. C with T). Six substitutions between a purine and a pyrimidine were discovered. In 198 position in $T$. axei and $T$. colubriformis, there was always a purine (A), whereas in $T$. probolurus, there was a pyrimidine (T) according to our data and a purine (G) according to Chilton et al. (1998).

The level of interspecific differences in the ITS-2 of Trichostrongylus spp. of our collection was at $3.5 \%$ for $T$. axei and $T$. probolurus, $3.0 \%$ for $T$. axei and $T$. colubriformis, and $3.9 \%$ for $T$. probolurus and T. colubriformis.

The dendrogram (Fig. 2) represents the similarity level among the ITS-2 sequences of three species of Trichostrongylus and the intraspecific isolates. It is possible to clearly mark only two clusters at the phylogenetic tree, $T$. colubriformis and $T$. probolurus. These two close species differ well, and their geographical isolates group closely with high bootstrap-support.

Another species, T. axei, particularly the Russian isolate, is characterized by relatively high level of interspecific and intraspecific differences.

\section{Discussion}

Sequence data of the ITS-2 rDNA for three Trichostrongylus spp. from the territory of Russia have been obtained for the first time. A comparison of the ITS-2 sequences of T. axei, T. colubriformis and T. probolurus from sheep from Moscow region revealed interspecific differences ranging from $3 \%$ to $4 \%$. When compared with the ITS-2 sequences of Trichostrongylus spp. from Australia and

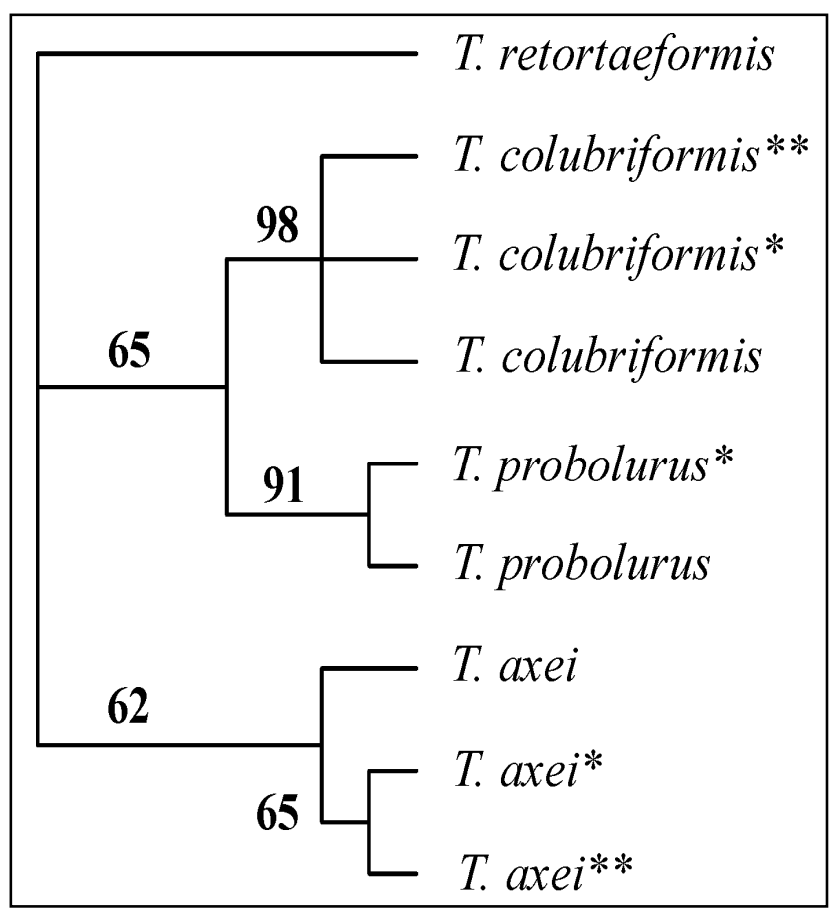

Fig. 2. Consensus tree depicting the ITS-2 similarity of three Trichostrongylus species according to our results, Hoste et al. (1995) for T. axei*, Hoste et al. (1993) for T. colubriformis*, Chilton et al. (1998) for T. probolurus*, Heise et al. (1999) for T. axei** and T. colubriformis**

Germany, the extent of the intraspecific variation ranged from $0 \%$ in $T$. colubriformis to $3.0 \%$ in $T$. axei.

Similar results were obtained in ITS-2 studies of Trichostrongylus spp. from ruminants collected in different geographic areas (Chilton et al., 1998; Heise et al., 1999; Hoste et al., 1993, 1995). Thus, differences between $T$. axei from bison and T. colubriformis from sheep from Australia made $3.4 \%$ (Hoste et al., 1995). The level of homology between $T$. axei and T. colubriformis from ruminants from Germany was at $95.0 \%$ (Heise et al., 1999). Hoste et al. (1995) noted the absence of the ITS-2 intraspecific variations in T. colubriformis, whereas Heise et al. (1999) registered variations at $1.26 \%$ level.

For analysis of intraspecific differences, comparisons were made between our data and previously published data for $T$. axei from bison and T. probolurus and T. colubriformis from sheep from Australia (Chilton et al., 1998; Hoste et al., 1993, 1995). The $0.9 \%$ differences of sequences in $T$. axei and $1.3 \%$ in T. probolurus were found. Intraspecific differences in the ITS-2 of $T$. axei from Russia (our data) and Germany (Heise et al., 1999) were found at $3.0 \%$ level. The same level of interspecific variations in the ITS-2 was found for T. axei and T. colubriformis (our data, Hoste et al., 1993) which exceeds slightly the differences between $T$. axei and $T$. probolurus from sheep from Australia. The dendrogram (Fig. 2) represents the high level of similarity between two geographic isolates of $T$. probolurus and lack of intraspecific differences in the ITS-2 of $T$. colubriformis. Relatively high genetic divergence among isolates of $T$. axei can be possibly explained by host-speci- 
ficity effect due to presence of intraspecific differences in the ITS-2 of $T$. axei parasitic in bison and in sheep. As Heise et al. (1999) did not give the species name of ruminants in Germany from which the material was obtained, it could not be decided whether host specificity effect was present.

Study of the ITS-2 sequences of three species of Trichostrongylus from Russia confirmed the low level of genetic differences within each of these species and provided additional information with respect to genetic variation within these parasites from different hosts and geographical regions.

\section{Acknowledgements}

This work was supported by the grant 05-04-48140a from Russian Foundation for Basic Research. The authors want to acknowledge E. S. Ivanova and S. E. Spiridonov for their critical comments and improvement of the English text.

\section{References}

Akbaev, M. Sh., Vodyanov, A. A., Kosminkov, N. E., Yatusevitch, A. I., Pashkin, P. I., Vasylevitch, F. I. (1998): Parasitology and invasive diseases of animals. Pub. House Kolos, Moscow

ASADOV, S. M. (1960): Fauna of helminthes of ruminants of USSR and analysis of its distribution and ecology. Pub. House of Azerbaijan SSR Academy of Sciences, Baku Boev, S. N., SoKolova, I. B., PANin, V. YA. (1963): Helminthes of ruminants of Kazakhstan. V. II. Pub. House of Kasakh SSR Academy of Sciences, Alma-Ata

Chilton, N. B., Hoste, H., Newton, L. A., Beveridge, I., GASSER, R. B. (1998): Common secondary structures for the second internal transcribed spacer pre-rRNA of two subfamilies of trichostrongylid nematodes. Int. J. Parasitol., 28: $1765-1773$

DAllas, J. F., IRvine, R. J., HALVORSEN, O. (2000): DNA evidence that Ostertagia gruehneri and Ostertagia arctica (Nematoda: Ostertagiinae) in reindeer from Norway and Svalbard are conspecific. Int. J. Parasitol., 30: 655 - 658

Gasser, R. B., Chilton, N. B., Hoste, H., Beveridge, I. (1993): Rapid sequencing of rDNA from single worms and eggs of parasitic helminths. Nucleic Acids Res., 21: 2525 2526

RECEIVED JUNE 20, 2006
Govorka, Ya., Maklakova, L. P., Mitukh, Ya., PelGunov, A. N., Rykovskyi, A. S., Semenova, M. K., SoNIN, M. D., ERKhardova-Kotrla, B., YurasheK, V. (1988): Helminthes of wild ungulates in Eastern Europe. Pub. House Nauka, Moscow

Heise, M., Epe, C., SchneIder, T. (1999): Differences in the second internal transcribed spacer (ITS-2) of eight species of gastrointestinal nematodes of ruminants. J. Parasitol., 85: $431-435$

Hoste, H., Gasser, R. B., Chilton, N. B., Mallet, S., BEVERIDGE, I. (1993): Lack of intraspecific variation in the second internal transcribed spacer (ITS-2) of Trichostrongylus colubriformis rDNA. Int. J. Parasitol., 23: 1069 1071

Hoste, H., Chilton, N. B., Gasser, R. B., Beveridge, I. (1995): Differences in the second internal transcribed spacer (ribosomal DNA) between five species of Trichostrongylus (Nematoda: Trichostrongylidae). Int. J. Parasitol., 25: $75-80$

Hoste, H., Chilton, N. B., Beveridge, I., Gasser, R. B. (1998): A comparison of the first internal transcribed spacer of ribosomal DNA in seven species of Trichostrongylus (Nematoda: Trichostrongylidae). Int. J. Parasitol., 28: $1251-1260$

IVAshkin, V. M., Oripov, A. O., Sonin, M. D. (1989): Keys to helminthes of small cattle. Pub. House Nauka, Moscow

McMurtry, L. W., Donaghy, M. J., Vlassoff, A., DouCH, P. G. C. (2000): Distinguishing morphological features of the third larval stage of ovine Trichostrongylus spp. Vet. Parasitol., 90: $73-81$

PryadKo, E. I. (1976): Helminthes of deer. Pub. House Nauka of Kazakh SSR, Alma-Ata

Skruabin, K. I., Shikhobalova, N. P., Shults R. S. (1954): Essentials of nematodology III. Trichostrongyloids of animals and man. Pub. House of Academy of Sciences USSR, Moscow

Skrjabin, K. I., Petrov, A. M. (1964): Essentials of veterinary nematodology. Pub. House Kolos, Moscow

SwOFFORD, D. L. (1998): PAUP*. Phylogenetic analysis using parsimony. Version 4. Sinauer, Sunderland, MA Wimmer, B., Craig, B. H., Pilkington, J. G., PemberTON, J. M. (2004): Non-invasive assessment of parasitic nematode species diversity in wild Soay sheep using molecular markers. Int. J. Parasitol., 34: 625 - 631

ACCEPTED FEBRUARY 19, 2007 